Journal of

Molecular Microbiology

\title{
Synthetic, Switchable Enzymes
}

\author{
Vic Norris ${ }^{a}$ Sergey N. Krylov ${ }^{b}$ Pratul K. Agarwal ${ }^{c, d}$ Glenn J. White ${ }^{e, f}$ \\ ${ }^{a}$ Theoretical Biology Unit, EA 4312, Department of Biology, University of Rouen, Mont Saint Aignan, France; \\ ${ }^{b}$ Department of Chemistry, York University, Toronto, ON, Canada; ${ }^{c}$ Computational Biology Institute and \\ Computer Science and Mathematics Division, Oak Ridge National Laboratory, Oak Ridge, TN, and d Department \\ of Biochemistry, Cellular and Molecular Biology, University of Tennessee, Knoxville, TN, USA; eSpace Science \\ and Technology Department, CCLRC Rutherford Appleton Laboratory, Didcot, and ${ }^{\mathrm{f}}$ Department of Physics and \\ Astronomy, The Open University, Walton Hall, Milton Keynes, UK
}

\section{Keywords}

Catalyst $\cdot$ Catalytic antibody $\cdot$ Ribozyme $\cdot$ Enzyme $\cdot$ Aptamer $\cdot$ Synthetic biology $\cdot$ Radiation · Sound · Light

\section{Abstract}

The construction of switchable, radiation-controlled, aptameric enzymes - "swenzymes" - is, in principle, feasible. We propose a strategy to make such catalysts from 2 (or more) aptamers each selected to bind specifically to one of the substrates in, for example, a 2-substrate reaction. Construction of a combinatorial library of candidate swenzymes entails selecting a set of a million aptamers that bind one substrate and a second set of a million aptamers that bind the second substrate; the aptamers in these sets are then linked pairwise by a linker, thus bringing together the substrates. In the presence of the substrates, some linked aptamer pairs catalyze the reaction when exposed to external energy in the form of a specific frequency of low-intensity, nonionizing electromagnetic or acoustic radiation. Such swenzymes are detected via a separate product-capturing aptamer that changes conformation on capturing the product; this altered conformation allows it (1) to bind to every potential swenzyme in its vicinity (thereby giving a higher probability of capture to the swenzymes that generate the product) and (2) to bind to

\section{KARGER}

(C) 2017 S. Karger AG, Basel

E-Mail karger@karger.com

www.karger.com $/ \mathrm{mmb}$ a sequence on a magnetic bead (thereby permitting purification of the swenzyme plus product-capturing aptamer by precipitation). Attempts to implement the swenzyme strategy may help elucidate fundamental problems in enzyme catalysis.

(c) 2017 S. Karger AG, Basel

\section{Introduction}

The modulation and control of enzyme functioning is one of the primary characteristics of biological systems. The Holy Grail for biotechnology would be a technique that could allow enzymes to be made easily - and, then, to be activated and inactivated at the flip of a switch. The ability to engineer a range of synthetic enzymes whose catalytic functions could be switched on and off by an external and controllable signal would facilitate many applications in sensing and therapeutics, and lead to new techniques that could be deployed in biotechnology. Two aspects of enzyme functioning might usefully be targeted: reaction rates and specificity for reactant and products.

A variety of types of molecules have been used to catalyze reactions. In pioneering studies, a molecular template, 3,5-Bis(5-[6-amino-3-benzyl-2-oxopyridyl])biphe-

\footnotetext{
Vic Norris

Theoretical Biology Unit, EA 4312, Department of Biology, University of Rouen FR-76821 Mont Saint Aignan (France)

E-Mail victor.norris@univ-rouen.fr
} 
nyl, was used to bind 2 substrates simultaneously to form a transient ternary complex thereby catalyzing the alkylation of an amine by an alkyl halide 6-fold [Kelly et al., 1990]. DNA has long been used as a template in reactions [Inoue et al., 1984; Naylor and Gilham, 1966; von Kiedrowski, 1986], for example, to form a complex with an alkyne and an alkene, thereby allowing palladium to catalyze production of an enone [Kanan et al., 2004]. In particular, antibodies developed in response to transition state analogs of targeted reactions have been seen as promising catalysts. However, although monoclonal antibodies that are able to carry out catalytic functions do occur in nature, they have never proven to be as efficient as enzymes in catalytic activity [Hilvert, 2000]. Despite early optimism and decades of intense research [Blackburn et al., 1989], the number of catalytic antibodies that have been obtained remains small. In 1994, it was conjectured that binding affinity alone might not be a useful way to select potential catalysts and that such candidates should be directly screened for their catalytic activity [Stewart et al., 1994]. More recently, it has been argued that to design such antibodies to catalyze a particular in vivo reaction requires that the catalytic mechanism itself first be understood [Zheng and Zhan, 2008] and, in 2011, it was observed that "attempts to design or synthesise molecules approaching the catalytic efficiency of enzymes have been unsuccessful thus far" [Hammes et al., 2011]. Unfortunately, biochemical engineering of enzymes de novo based on a number of different approaches has proven to be hard work [Preiswerk et al., 2014].

The problem is compounded by the fact that whilst "understanding the incredible catalytic efficiency of enzyme catalysis in molecular terms has been a goal of biochemistry for over half a century," this understanding remains elusive [Hammes et al., 2011]. One productive approach has been to consider enzyme catalysis as "progress over a multi-dimensional energy landscape where ensembles of interconverting conformational substates channel the enzyme through its catalytic cycle" [Boehr et al., 2010]. In this approach, enzymes have conformations with population distributions that change as the catalytic cycle proceeds [Ramanathan et al., 2014]. In the case of aspartate aminotransferase, which catalyzes the transfer of an amino group from aspartate to ketoglutarate to give oxaloacetate and glutamate, 15 reaction intermediates have been identified for the overall reaction mechanism.

These interconverting conformations arise because an enzyme undergoes a wide range of spatial and temporal scales of internal motions; those involving bond vibrations and fluctuations within a group of a few atoms take femtoseconds to picoseconds whilst those involving collective fluctuations of subdomains or the entire protein take milliseconds [Agarwal, 2006; Cannon and Benkovic, 1998; Henzler-Wildman and Kern, 2007]. These fluctuations may be coupled in a complex organized hierarchy of levels that differ from one another energetically such that, within a set of substates of conformational fluctuations, the substates are separated by small energy barriers from each other whilst the sets themselves are separated from one another by a larger energy barrier [Benkovic et al., 2008; Boehr et al., 2006]. In this approach, conformational fluctuations and transitions between multiple substates are central not only to enzyme catalysis [Boehr et al., 2006; Fraser et al., 2009], but also to molecular recognition [Lange et al., 2008; McHaourab et al., 1997; Ramanathan and Agarwal, 2009].

The roles of conformational changes in catalysis are exemplified by the hydride transfer reaction catalyzed by dihydrofolate reductase where, in one theoretical approach called empirical valence bond, a collective reaction coordinate can be defined in terms of the energy difference between the reactant and product valence bond states. The results from this approach show that the reaction involves movements of enzyme, substrate and cofactor such that conformational changes along the reaction coordinate bring the donor and acceptor closer, orient the substrate and cofactor, and create an appropriate electrostatic environment. It is believed that the time needed for hydride transfer depends on the millisecond times needed to sample those conformations that can permit the reaction [Hammes-Schiffer and Benkovic, 2006; Hammes et al., 2011]. The likely explanation is that the free energy barrier to the actual chemical bond breaking and allowing the reaction is the low probability of sampling conformations at the top of the barrier relative to the large number of those conformations at the bottom of the barrier. In the quest for synthetic enzymes, a fundamental question is, therefore, how the probabilities of sampling conformations might be manipulated.

A conformational change is often associated with the binding of the reactants at the start of catalysis. This change then leads to changes at the active site including the exclusion of solvent water, the reduction of the dielectric constant, and alteration of the $\mathrm{p} K_{\mathrm{a}}$ [Hammes et al., 2011]. There are several ways to obtain oligonucleotides or peptides that undergo conformational changes on binding ligands. Oligonucleotide aptamers that undergo conformational changes upon binding ligands are an attractive choice as they can be generated in vitro. In particular, there is a capillary-electrophoresis-based 
technology for highly efficient selection of oligonucleotide aptamers [Krylov, 2007]. It requires very few rounds of selection and, as a result, generates highly diverse, enriched libraries that probably contain around a million different sequences of aptamers [Kanoatov et al., 2010].

One route to obtaining enzymes to order might be to manipulate the conformations of macromolecules via exposure to nonionizing electromagnetic radiation or to acoustic radiation. For example, exposure of lipases to ultrasound at $40 \mathrm{kHz}$ changed their tertiary structures and led to an increased catalytic activity [Shah and Gupta, 2008]; exposure of $\beta$-lactoglobulin to ultrasound also altered its structure [Stanic-Vucinic et al., 2012], and exposure to laser pulses has been shown to excite an enzymesubstrate complex, inducing a favorable conformation of the active site, and directly enabling the coupled hydride and proton transfer reactions to occur [Sytina et al., 2008]. Exposure to electromagnetic radiation in the $\mathrm{GHz}$ range is also likely to influence protein conformations given that a variety of $\mathrm{GHz} / \mathrm{THz}$ frequencies have been calculated for the $\alpha$-helices of the catabolite-gene activator protein, CAP [Volkov and Kosevich, 1991]. Such radiation could affect interactions between molecules and the surrounding matrix, as has already been shown in the case of the release of ethylene oxide from a surface following exposure to low intensity radiation at $2.45 \mathrm{GHz}$ which disrupts hydrogen bonds in a nonthermal fashion [Gibson et al., 1988]. Exposure of lysosome to visible and UV light in the presence of a light-responsive surfactant has been used to reversibly increase and decrease the activity (and corresponding structures) of the enzyme [Wang and Lee, 2007]. Exposure of the $\beta$-glucosidase, CelB, from a hyperthermophilic organism to microwave radiation allowed this enzyme to catalyze its reaction far below its thermal optimum because, the authors suggest, conformational flexibility was induced by a rapid dipole alignment of the peptide bonds with the oscillating electric field [Young et al., 2008]. Recently, the heat released during catalysis by an enzyme has been shown to affect its diffusion [Riedel et al., 2015] via, the authors propose, a process similar to that occurring in photoacoustic spectroscopy in which a vibrationally excited protein relaxes by dissipating its energy into the solvent through acoustic waves [Peters and Snyder, 1988]. Finally, selective enzyme conformations have been altered by external manipulation using chemical modifications to the surface of the enzyme as in the case of the light-activated molecular switch on the surface of lipase B that enhances catalysis [Agarwal et al., 2012]. Note that in this approach the pho-

Synthetic, Switchable Enzymes tons from light do not directly change the conformational equilibria through stochastic collisions, but the energy of the photons is converted to mechanical energy by driving the cis-trans isomerization on the $\mathrm{N}=\mathrm{N}$ bond in the azobenzene. This mechanical energy is hypothesized to alter the conformational equilibria such that more enzyme conformations reach the functionally relevant conformation substates. This is similar to recent evidence where the motions of the solvent surrounding the enzyme surface can alter functional enzyme dynamics [Duff et al., 2017].

Even if synthetic enzymes can be created and induced to function, there may well be the problem of selecting them from a much larger population of nonfunctional candidates. Here, however, it should be possible to profit from the elevated concentration of products around the enzyme that catalyzes the reaction by using another aptamer designed to bind to the neighboring enzyme only when this product aptamer has bound the product.

In what follows, we propose a strategy to obtain synthetic, switchable, enzymes - which we term "swenzymes" - based upon nucleic acid aptamers and exposure to either electromagnetic or acoustic radiation. We then discuss the problems associated with this strategy and consider possible solutions. We also discuss how pursuit of the goal of swenzymes may help elucidate the mechanism of enzyme catalysis and other problems even if the goal itself turns out to be unattainable.

\section{Principle of Swenzyme Catalysis}

Four conceptual steps are involved in obtaining swenzymes capable of catalyzing a reaction with 2 substrates (where "substrate" is used in the biochemical sense of "reactant") that yields one product: (1) selecting the binders (macromolecules that bind to the substrates), (2) ligating the binders to make candidate swenzymes, (3) stimulating these candidates by exposure to radiation, and (4) selecting the candidates that actually catalyze the reaction.

\section{Selecting the Binders}

An aptamer (i.e., a macromolecule) is selected to bind strongly to one of the substrates. Another macromolecule is selected to bind strongly to the other substrate.

\section{Ligating the Binders}

Each of the binders to substrate 1, the 1-binders, is ligated to each of the binders to substrate 2, the 2-binders, so as to produce candidate swenzymes.

J Mol Microbiol Biotechnol 2017;27:117-127 DOI: $10.1159 / 000464443$ 
Fig. 1. The principle of the swenzyme. a From a large library of aptamers, an aptamer, $\mathrm{S1}^{\prime}$, is obtained to bind to substrate $1, \mathrm{~S} 1$, and a different aptamer, S2', is obtained to bind to substrate 2, S2. b A linker joins the two aptamers covalently so as to obtain a candidate swenzyme that can bind simultaneously to both substrate 1 and substrate 2 . c The candidate swenzyme is exposed to different frequencies of radiation (red lightning) so as to allow it to catalyze the reaction.

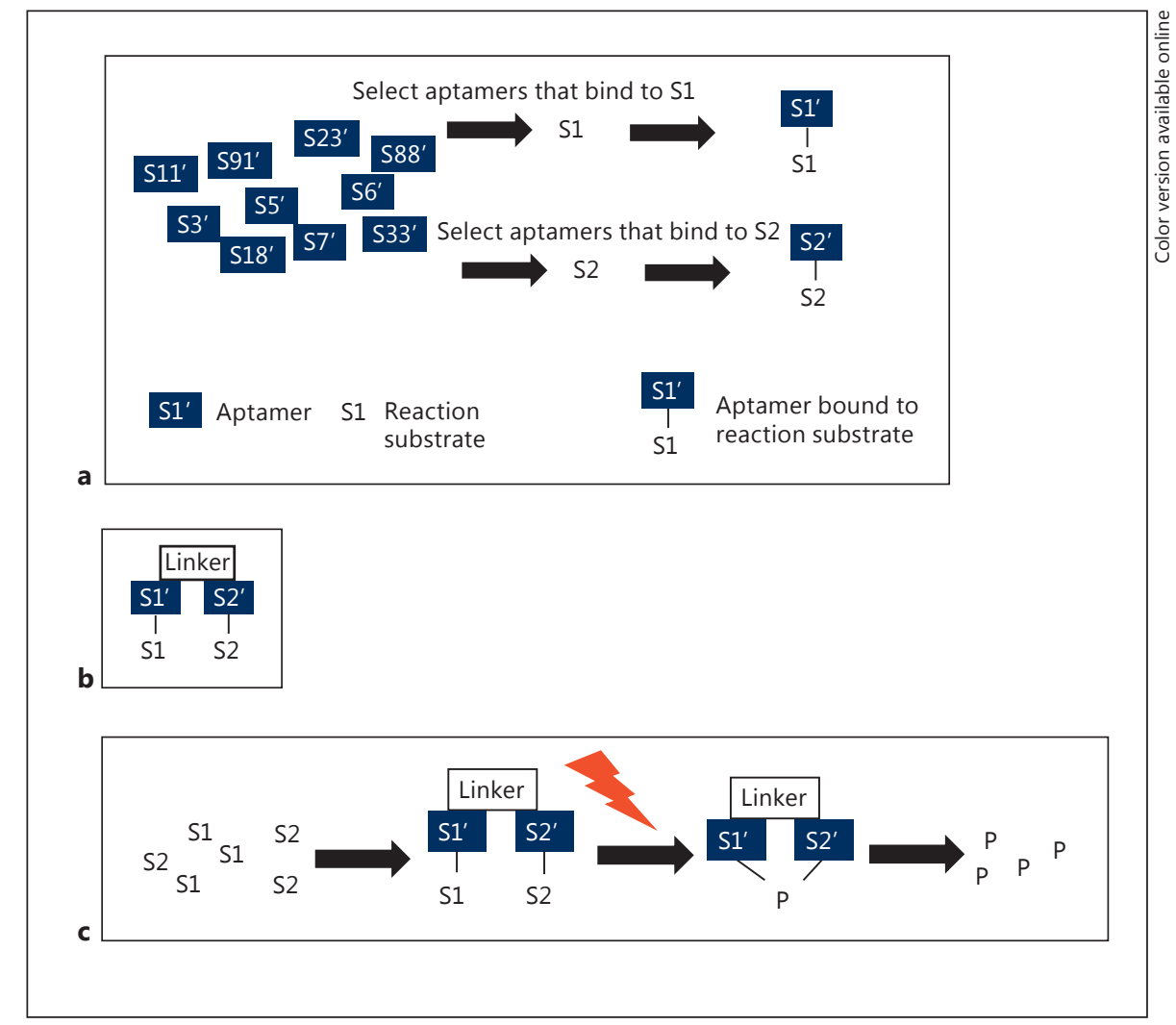

\section{Stimulating the Candidate Swenzymes}

Each of the covalently linked binder pairs - the candidate swenzymes - is tested separately for its capacity to catalyze the reaction whilst it undergoes conformational fluctuations that are stimulated by exposure to different frequencies of low-intensity radiation

\section{Detecting Swenzymes}

The swenzymes are detected by the decrease in substrates or the increase in product.

\section{Initial Implementation Strategy}

\section{Construction of the Binder Libraries}

The macromolecules that constitute the binders may be polynucleotides, polypeptides, polysaccharides, etc. In the implementation described here, the 1-binder library is obtained separately from the 2-binder library. The binders should have the "right" characteristics of specificity and affinity so that they bind to the substrates when these are present in low concentrations in a complex mixture, and yet release the product which may contain the same epitopes as the substrates themselves. Aptamers made of single-stranded DNA can provide the required structural diversity and a range of affinities required for choosing molecules with the desired binding properties. There are also strategies that allow highly-diverse libraries of DNA aptamers for substrates, which should be of benefit in constructing swenzymes [Yufa et al., 2015].

\section{Ligation}

In the implementation described here, a single 1-binder is ligated to a single 2-binder to make a 1,2-binder (see below). Ligation may be direct, but it may be more effective to use a linker. The linker itself is made of DNA and may have been designed to have particular characteristics. For example, it may explore a conformational space in which it spends part of the time as a hairpin-like structure that brings together its ends; the design of such structures is relatively straightforward in the case of oligonucleotides since they result from base complementarity.

\section{Selection of Candidate Swenzymes}

The candidates are exposed to low-intensity, nonionizing, electromagnetic radiation or acoustic radiation at a 


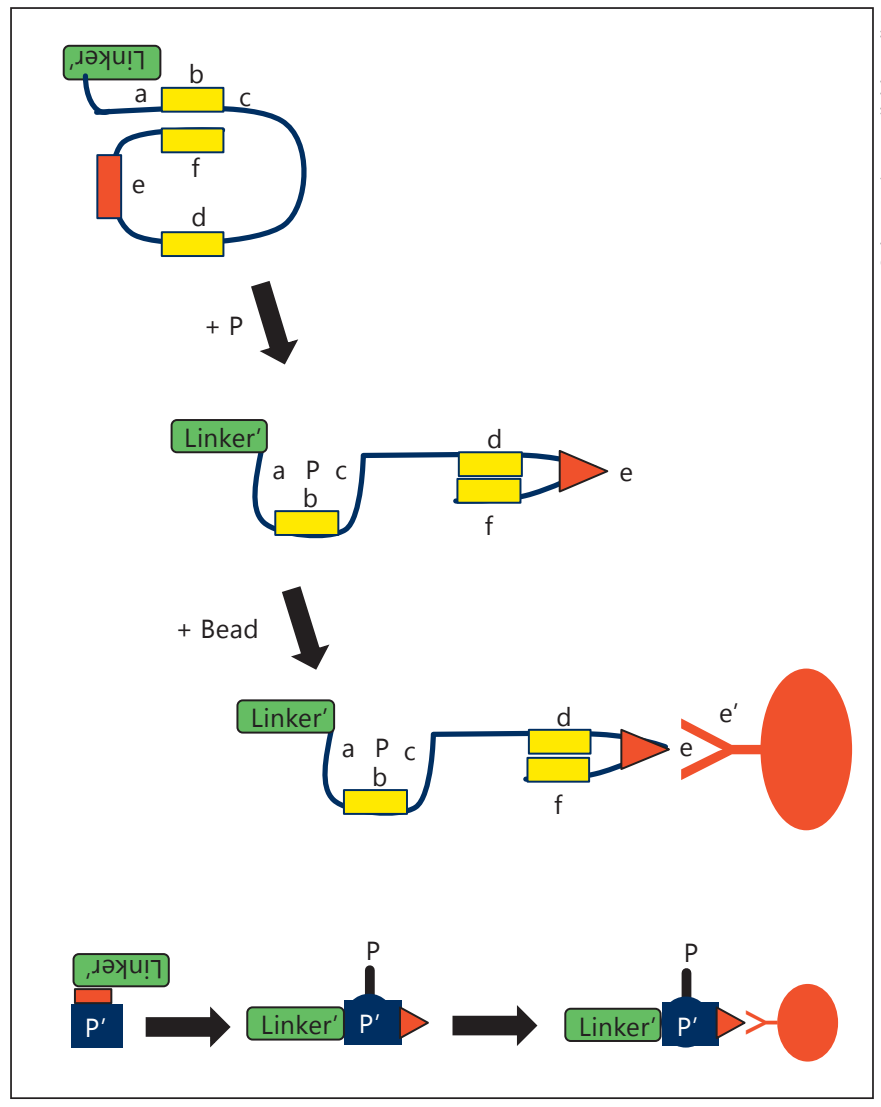

Fig. 2. Principle of the aptameric detector of product. The idea is to detect indirectly the presence of the reaction product, $\mathrm{P}$, because $\mathrm{P}$ binds to an aptamer, $\mathrm{P}^{\prime}$, thereby changing the conformation of this aptamer, and it is this changed product aptamer that is then detected. In the absence of product, $\mathrm{P}$, nucleotide sequences $\mathrm{b}$ and $\mathrm{f}$ bind one another and $\mathrm{d}$ is unbound. In the presence of $\mathrm{P}$, which binds $\mathrm{a}, \mathrm{b}$, and $\mathrm{c}$, the binding of $\mathrm{b}$ and $\mathrm{f}$ is disrupted; this allows $\mathrm{d}$ to bind $f$ and thereby (1) change the conformation of the linker' in green that binds to the linker in a nearby candidate swenzyme (this is why linker' goes from upside-down to right-way-up) and (2) change the conformation of e to one that can be recognized by the sequence $\mathrm{e}^{\prime}$, which is attached to a magnetic bead (large red oval). The complex sketches are represented by the simple symbols at the bottom of the figure.

wide range of frequencies. As a starting point, this exposure would take the form of a stepping through the frequencies, focusing on those characteristic of the largescale conformational changes in the linker (or chassis, see below) where this is used. During this exposure, candidate swenzymes are selected in the presence of the substrates for those that catalyze the reaction (Fig. 1). Although such screening could be based on the individual characteristics of the substrates and products in a particular reaction, we propose that it be based on a general strat-

Synthetic, Switchable Enzymes

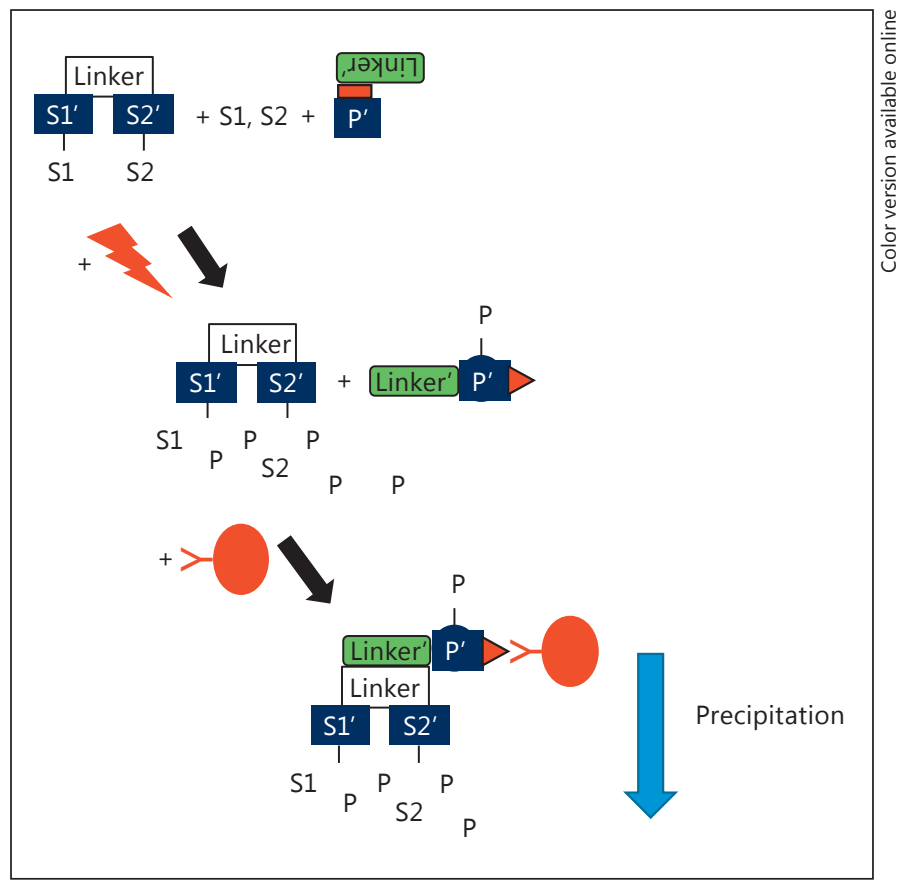

Fig. 3. The process of selection of a swenzyme. A viscous mixture of candidate swenzymes, substrates, product detectors, and magnetic beads is exposed to radiation. The swenzyme-catalyzed reaction leads to a locally high concentration of the products around the swenzyme. These products cause the product detector to change conformation and to bind to both the neighboring swenzyme and a sequence on a magnetic bead. The magnetic bead then allows the ensemble of aptamers in the vicinity of the product aptamer (which contains the swenzyme itself) to be precipitated. Symbols as in Figures 1 and 2.

egy. This strategy could, for example, entail the use of aptamers selected to bind to an epitope in the product that would be absent from the substrates. For example, a product-capturing DNA sequence is part of an aptamer that changes conformation on a binding product so as to both bind potential swenzymes in its vicinity and be bound itself by a sequence attached to a magnetic bead (Fig. 2). This "belt-and-braces" approach maximizes the chances of coprecipitation of the swenzyme, productcapturing aptamer, and bead (Fig. 3).

\section{Theoretical Considerations: Energetic Analysis for Enzyme Catalytic Efficiency}

An important theoretical consideration when developing designer enzymes concerns their catalytic efficiency, particularly the reaction kinetics. Recently, de novo 
approaches have been used to design proteins to serve as enzymes, but these proteins typically exhibit $10^{5}-10^{6}$ fold less catalytic efficiency than the corresponding natural enzymes [Siegel et al., 2010]. It is widely discussed that enzymes can speed up reactions by $>10^{20}$ compared with reactions without enzymes, and it has further been suggested that half of this contribution comes from the structural environment and electrostatic stabilization. However, there is an activation barrier that needs to be crossed by these enzymes, which manifests itself as the rate of enzyme reaction $\left(k_{\text {cat }}\right)$. Therefore, to improve the catalytic efficiency $10^{5}-10^{6}$-fold, strategies could be designed to provide additional energy to overcome the activation energy barrier. Using the transition state theory framework, we can estimate the energy required to be provided for the designer enzymes. For the enzymatic reaction rate constant (in the TST framework) $k_{\mathrm{TST}}$ we have:

$$
k_{\mathrm{TST}}=\frac{k_{\mathrm{B}} T}{h} e^{-\frac{\Delta G}{k_{\mathrm{B}} T}},
$$

where $k_{\mathrm{B}}$ is the Boltzmann constant, $h$ is Planck's constant, $\Delta G$ is the activation energy barrier, and $T$ is the temperature. Using this equation, we can calculate roughly the amount of energy that would be required. In ambient conditions of $300 \mathrm{~K}$, a roughly 10 -fold increase in rate constant corresponds to a $5.73-\mathrm{kJ} / \mathrm{mol}(1.37 \mathrm{kcal} / \mathrm{mol})$ lowering in energy of activation. Hence, a $10^{5}-10^{6}$-fold improvement would correspond to $28.8-34.3-\mathrm{kJ} / \mathrm{mol}$ $(6.9-8.2 \mathrm{kcal} / \mathrm{mol})$ extra energy requirement to cross the activation energy barrier. It is worth noting that the energy required need not be supplied by a single photon as enzymatic reactions have several barriers, each requiring a lower energy, which can be overcome sequentially by supplying many photons of lower energy.

More realistically, even moderate improvements of $10^{2}-10^{3}$-fold would be achievable with only $11.7-13.4 \mathrm{~kJ} /$ $\mathrm{mol}(2.8-3.2 \mathrm{kcal} / \mathrm{mol})$ of extra energy. Consider for example, the strategy we used in photoactivated conformational modulation [Agarwal et al., 2012]. The design is based on driving the conformations of protein by activating an azobenzene linker with UV light (with peak wavelength at $360 \mathrm{~nm}$ ), which corresponds to photons of 334 $\mathrm{kJ} / \mathrm{mol}(\sim 80.0 \mathrm{kcal} / \mathrm{mol})$. As a result of using this strategy, a roughly 30 -fold improvement in enzyme activity was achieved. This 30 -fold improvement roughly corresponds to $8.4 \mathrm{~kJ} / \mathrm{mol}(2 \mathrm{kcal} / \mathrm{mol})$ energy supplied to the reaction coordinate along the enzyme reaction pathway. Therefore, strategies that convert photochemical energy into mechanical energy to overcome the activation barrier would be useful in improving the catalytic efficiency of designed enzymes.

Microwave radiation has also been used to accelerate catalysis [Young et al., 2008]. One potential advantage of using microwave radiation lies in the absence of a requirement for any specific modification to the enzymes, whilst a disadvantage is that the energy associated with microwave radiation is much lower than at shorter wavelengths. Nonetheless, strategies that allow external sources of energy to aid enzyme functioning would be useful in improving the catalytic efficiency of the overall process.

\section{Anticipated Problems and Possible Solutions}

\section{Selection of Binders to Small Molecules}

If the separation between the aptamers and aptamers bound to the substrates is insufficient to permit selection, tagged substrates can be used. This may take the form of a small macromolecular tag such as polyethylene glycol or a peptide which facilitates separation [Krylov, 2007; Wegman et al., 2013]. In this case, there is the danger of selecting aptamers that bind only to the tag; a possible solution would be to purge the library from which the binders are obtained by discarding all those aptamers that bind to the tag on its own.

\section{Ligation}

In the initial construction of the aptamers, a sequence is added to each aptamer that would allow it to be ligated. For example, the 1-binders have a sequence at their $3^{\prime}$ end complementary to a sequence at the end of the 2-binders; this would permit the 1-binders to be ligated only to the 2-binders.

\section{Stimulation of Candidate Swenzymes}

What frequencies of radiation should be used to stimulate the reaction? One can step progressively through the frequencies, testing the effects of one at a time, but what if the 1-binders and the 2-binders change conformations in response to exposure to different frequencies? As discussed above, one possibility would be to first select the aptamers binding to the substrates as above and then to rerun the experiment with these 1-binders and 2-binders again in the presence of the substrates; in the rerun, the binders would be exposed to radiation and this time the aptamers would be selected that had released the substrates. This approach would therefore favor selection of the class of binders with bonds to the substrates that are distorted on irradiation and it might be argued that this 
is the exactly the class that is needed to produce the transition state intermediate.

A complementary possibility would be to use an already characterized structure of protein or ribozyme as a preexisting chassis (this would also take into account the fact that despite numerous attempts to reduce the size of enzymes, the minimum size for efficiency seems to be 10 $\mathrm{kDa}$ [Hammes et al., 2011]. The conformational space of such a chassis might already have been determined as well as, in the ideal world, the frequencies with which this chassis changes conformation. If a hairpin RNA or singlestranded DNA aptamer were used as the chassis, the 1and 2-binder sequences could be ligated into the loop even before selection for binding (see below). One advantage to the use of a relatively large chassis is that its exploration of conformational changes may organize the frequencies of its constituent structures. In other words, the chassis may impose coherence by coupling the oscillations of the 1-and 2-binder sequences with the result that the 1- and 2-binder sequences joined by a chassis would tend to oscillate with the same frequency [Strogatz and Stewart, 1993].

Finally, if several fluctuations and frequencies of fluctuations are needed for catalysis by a swenzyme, the use of a chassis with a characterized conformational space may give a pointer as to which combinations of frequencies should be used. Indeed, in the case of the computational design of enzymes, the rigidity of the chassis or scaffold is an important factor [Preiswerk et al., 2014].

\section{Detection}

Reducing the diffusion of products so as to increase the probability of precipitating those swenzymes that have catalyzed the reaction may be insufficient to prevent precipitation of an excessive number of false positives. Alternative strategies to give a higher probability of capture to the swenzymes that generate the product may be based on incorporating a product-capturing, conformation-changing, DNA sequence into the sequences of the potential swenzymes themselves; this sequence in its product-bound state would then be recognized by the precipitation system.

A very different detection system might be based on using a 2-D sandwich of an array of candidate swenzymes and a second array of aptameric, product detectors; if the substrates were labeled with different stable isotopes like ${ }^{13} \mathrm{C}$ and ${ }^{15} \mathrm{~N}$, the technique of dynamic secondary ion mass spectrometry might be used to detect the presence of the ${ }^{13} \mathrm{C}^{15} \mathrm{~N}^{-}$recombinant secondary ion in the product [Lechene et al., 2007; Legent et al., 2008] and thereby allow

Synthetic, Switchable Enzymes the positions of the products detected on the second array to be mapped to the positions of the swenzymes on the first array.

\section{The Chassis Approach}

A more refined version of the swenzyme might be based on the use of a chassis. In choosing a chassis, use might be made of an RNA that forms a stem loop and that exhibits a reproducible range of coherent fluctuations that can be influenced by exposure to nonionizing electromagnetic radiation (or perhaps even to acoustic radiation). The chassis is encoded by DNA and the loop has 3 sites into which other DNA sequences can be cloned corresponding to the substrates and cofactor. The chassis also has a sequence at the $5^{\prime}$ end that is complementary to a sequence at the $3^{\prime}$ end in order to allow multimeric swenzymes to form.

The libraries from which the binders will be selected are cloned separately into the chassis, and RNA is made and purified. The binders are then selected during exposure to radiation at similar frequencies.

\section{Choice of Reaction for Pilot Study}

Initially, it would be sensible to try to catalyze a unimolecular reaction. Candidate reactions include Kemp elimination, which has been widely used to study the catalysis of proton removal from a carbon-hydrogen bond in the substrate; this catalysis, which is usually limited by high activation-energy barriers, requires as a minimum the presence of a base, and the reaction can be accelerated by catalysts as varied as synthetic compounds, catalytic antibodies, and serum albumin [Hu et al., 2004]. Combining computational enzyme design and molecular evolution has allowed synthetic enzymes to be generated to catalyze Kemp elimination at rates $>10^{6}$ of the uncatalyzed rate [Rothlisberger et al., 2008] and, more recently, at rates $>10^{8}$ [Blomberg et al., 2013]. Note that in Kemp elimination, cleavage of the substrate, 5-nitrobenzisoxazole, can readily be assayed in vitro by monitoring the formation of the product, salicylonitrile, spectroscopically at $380 \mathrm{~nm}$ [Blomberg et al., 2013].

The Diels-Alder reaction, which is often used by chemists to construct compounds, is another possibility. This reaction is a $[4+2]$ cycloaddition between a conjugated diene and a substituted alkene (or dienophile) to form a substituted cyclohexene system via a single, cyclic transi-

J Mol Microbiol Biotechnol 2017;27:117-127 
tion state with no intermediates generated during the reaction [Dewar et al., 1986]. Note that, in the case of ribozyme catalysis, this reaction can be monitored in vitro by fluorescence via probes that connect an anthracene diene with a substituted BODIPY dye [Gaffarogullari et al., 2015].

\section{Choice of Candidate Swenzymes for a Pilot Study}

As an initial exploration of the nature and frequency of radiation likely to affect catalysis, it may be worth trying to modify the efficiencies of existing catalysts. Good candidates include the catalytic antibodies used to catalyze Kemp elimination such as antibody $34 \mathrm{E} 4$, which has an efficiency reduced by conformational isomerism and reliance on a single catalytic residue, and antibody 13G5, which promotes selective cleavage of 6-glutaramidebenzisoxazole - an unactivated substrate - with multiple turnovers and rate accelerations $>10^{5}$ over background [Debler et al., 2009]. Candidate catalytic antibodies also include those that catalyze the Diels-Alder reaction [Hilvert et al., 1989; Marti et al., 2008; Romesberg et al., 1998]. Candidate ribozymes also exist and include a set of artificial ribozymes that catalyze the Diels-Alder reaction and that have already been characterized [Ameta et al., 2014]

\section{Discussion}

Rather than a piecemeal approach to studying enzyme mechanisms, the integrative strategy we propose brings together a variety of different concepts and experimental possibilities. Although the objective of creating synthetic, switchable enzymes - or swenzymes - may be difficult or even unattainable, the implementation of a strategy to obtain them may provide a framework for coordinating research into different problems related to enzyme catalysis.

One of these problems is whether there is a direct, dynamic coupling between the fast (picosecond to nanosecond) thermal motions (or indeed vibrations at millisecond frequencies) that bring the reactants, donor and acceptor together and the chemical reaction itself. For example, the rates of conformational changes in the reaction cycle control ligand release, as well as the chemical step. Possible mechanisms that might couple and coordinate the fluctuations at the different levels include water structure [Bellissent-Funel et al., 2016; Pollack, 2013; Sharma et al., 2013] and Manning-Oosawa ion condensa- tion [Manning, 2007; Musheev et al., 2013; Oosawa, 1971; Ripoll et al., 2004; Volker and Breslauer, 2005].The testing of such candidate mechanisms would be facilitated by the swenzyme scenario we propose.

Another problem is why so many reactions are catalyzed by proteins rather than polynucleotides. Quantitative information on the fundamental reasons for this may result from trying to construct swenzymes for the same reaction out of both polynucleotides - RNA, singlestranded DNA, and double-stranded DNA - and polypeptides; for example, it may prove possible to quantify the probabilities of obtaining catalysts from each of these classes of constituents.

A third problem or set of problems is whether it is better to catalyze reactions in vitro or in vivo. Swenzymes may be constructed to operate not only in vitro but also in vivo. Cloning the genes that encode swenzymes onto plasmids may allow their overproduction in vivo via either increased plasmid copy numbers in a temperaturesensitive system (for DNA-based swenzymes) or an induction of expression system (for RNA-based or peptidebased swenzymes). Although catalysis inside a cell raises the problem of the entry of the substrates and the secretion of the products, swenzymes could be expressed on the surface of bacteria as in the case of the cellulose which catalyzes reactions outside the cell [Doi and Kosugi, 2004]. This would be a step towards the construction of an entire inside-out metabolism [Norris et al., 2011].

A fourth problem is how to initiate or stop a reaction within cells. Techniques that allow such actions in vivo with minimal perturbation are of use in a wide variety of studies including those of differentiation and the cell cycle. Exposure to acoustic radiation or to nonionizing electromagnetic radiation that switched on the catalysis by an intracellular swenzyme would be a potent additional technique.

Finally, a problem to which synthetic biology may contribute is how to develop new methods of computing. We have argued that one of the fundamental characteristics of living systems is that they exhibit competitive coherence. This process entails the system trying to obtain a global coherence by reconciling its constrained behavior over time (i.e., a historical coherence) with its constrained behavior in the present state (i.e., environmental coherence). The process of competitive coherence can be thought of as a form of calculation that can result in learning [Norris et al., 2012]. The different trajectories an enzyme follows in conformational space reflect both its history and its present environment. It might therefore be argued that an enzyme is also displaying competitive co- 
herence and, in this sense, is a unit of calculation. Our swenzyme scenario may therefore eventually pave the way to new sorts of calculation, such as "bioputing" [Norris et al., 2011].

\section{Acknowledgement}

We thank Steve Benkovic for several very helpful comments, including suggesting which reactions we should focus on, and the anonymous reviewers for substantially improving the paper. P.K.A.'s effort was supported by a grant from National Institute of General Medical Sciences (NIGMS) of the National Institutes of Health (NIH) USA under award number R01GM105978. S.N.K.'s contribution to this work was supported by grant CPG 134747 from the Canadian Institutes of Health Research.

\section{Disclosure Statement}

The authors have no conflicts of interest to declare.

\section{Appendix}

Other Possible Approaches

Adding a Cofactor

A cofactor is a nonprotein chemical compound that is bound to a protein and is required for the biological activity of the protein. Loosely bound cofactors are sometimes termed "coenzymes" and tightly bound cofactors are sometimes termed "prosthetic groups." Examples of cofactors include thiamine pyrophosphate, lipoamide, flavin adenine dinucleotide, nicotinamide adenine dinucleotide, coenzyme A, biotin, tetrahydofolate, pyridoxal phosphate, and the ions of metals such as iron, magnesium, manganese, cobalt, copper, zinc, and molybdenum. The contribution of cofactors to catalysis might be evaluated if sequences binding to cofactors were to be selected and these sequences were then to be incorporated into the candidate swenzymes.

\section{Using Alternatives to Smart Aptamers}

Several systems could be used to construct the 1-binders, 2-binders, and the 1,2-binders, i.e., the candidate swenzymes [Tonelli et al., 2012]. Phage display [Smith, 1985] could be used with, for example, bacterial plaques (in which bacteriophages are concentrated) on a lawn of bacteria being transferred to nitrocellulose and probed with the product-binder to select swenzymes.
The binders might also be obtained from DNA and RNA aptamers selected via rounds of selection and amplification using SELEX (the Systematic Evolution of Ligands by Exponential Enrichment) [Takahashi et al., 2003; Yonezawa et al., 2004]. Ribosome display would be another possibility [He and Taussig, 2002].

Constructing Enzyme Hyperstructures

There are probably many reasons why the enzymes in modern cells associate with either the cytoskeleton or other enzymes, or indeed themselves [Ingerson-Mahar et al., 2010; Narayanaswamy et al., 2009; Norris et al., 1996; Weart et al., 2007]. These reasons include metabolic efficiency and signal transduction [An et al., 2008; Norris et al., 2013; Srere, 1994]. One possibility is that by associating with other elements in a higher order structure, the conformational space is significantly limited and catalytic activity thereby enhanced. To explore this possibility, the aptamers could be constructed with complementary sequences in order to favor their binding to one another to form linear hyperstructures.

Using an Alternative to Magnetic Beads for the Detection Step

One possibility would be to fuse the product detector (Fig. 2) to the sensor based on Spinach, a 98-nucleotide RNA sequence that binds a small-molecule fluorophore 3,5-difluoro-4-hydroxybenzylidene imidazolinone and that activates its fluorescence [Paige et al., 2012]; in this case, the binding of the product to the product detector would allow both (1) the product detector to bind to the swenzyme and (2) Spinach to interact with and activate the fluorophore; fluorescent swenzymes might then be selected using flow cytometry (note that an alternative scenario can be devised in which the candidate swenzymes themselves have a sequence to bind the product, change conformation, and activate the fluorophore). Kinetic capillary electrophoresis itself might be used to select swenzymes from candidate swenzymes [Yufa et al., 2015]. Suppose the candidates have bound the substrates, they can then be selected along with the bound substrates and remigrated. During this migration, they are exposed to radiation and those that catalyze the reaction and release the product migrate differently and can be selected (of course, some may simply release the substrates). This procedure would be facilitated if the substrates were tagged. Potentially better still would be to have a positive selection in vivo; for example, if the substrates were to diffuse into - or be transported into - bacteria containing the candidate swenzymes, the presence of the product could be used to induce the expression of a gene to suppress the conditional lethality resulting from a temperature-sensitive phenotype. Finally, these approaches could be complemented by using aptamers to epitopes in the substrates that are absent from the product so as to detect the lowering of substrate concentration as the reaction proceeds.

\section{References}

Synthetic, Switchable Enzymes
Agarwal PK: Enzymes: an integrated view of structure, dynamics and function. Microb Cell Fact 2006;5:2.

Agarwal PK, Schultz C, Kalivretenos A, Ghosh B, Broedel SE Jr: Engineering a hyper-catalytic enzyme by photoactivated conformation modulation. J Phys Chem Lett 2012;3: $1142-1146$.
Ameta S, Winz ML, Previti C, Jaschke A: Nextgeneration sequencing reveals how RNA catalysts evolve from random space. Nucleic Acids Res 2014;42:1303-1310.

An S, Kumar R, Sheets ED, Benkovic SJ: Reversible compartmentalization of de novo purine biosynthetic complexes in living cells. Science 2008;320:103-106 
Bellissent-Funel MC, Hassanali A, Havenith M, Henchman R, Pohl P, Sterpone F, van der Spoel D, Xu Y, Garcia AE: Water determines the structure and dynamics of proteins. Chem Rev 2016;116:7673-7697.

Benkovic SJ, Hammes GG, Hammes-Schiffer S: Free-energy landscape of enzyme catalysis. Biochemistry 2008;47:3317-3321.

Blackburn GM, Kang AS, Kingsbury GA, Burton DR: Catalytic antibodies. Biochem J 1989;262: 381-390.

Blomberg R, Kries H, Pinkas DM, Mittl PR, Grutter MG, Privett HK, Mayo SL, Hilvert D: Precision is essential for efficient catalysis in an evolved Kemp eliminase. Nature 2013;503: 418-421.

Boehr DD, McElheny D, Dyson HJ, Wright PE: The dynamic energy landscape of dihydrofolate reductase catalysis. Science 2006;313: 1638-1642.

Boehr DD, McElheny D, Dyson HJ, Wright PE: Millisecond timescale fluctuations in dihydrofolate reductase are exquisitely sensitive to the bound ligands. Proc Natl Acad Sci USA 2010;107:1373-1378.

Cannon WR, Benkovic SJ: Solvation, reorganization energy, and biological catalysis. J Biol Chem 1998;273:26257-26260.

Debler EW, Muller R, Hilvert D, Wilson IA: An aspartate and a water molecule mediate efficient acid-base catalysis in a tailored antibody pocket. Proc Natl Acad Sci USA 2009;106: 18539-18544.

Dewar MJ, Olivella S, Stewart JJ: Mechanism of the Diels-Alder reaction: reactions of butadiene with ethylene and cyanoethylenes. J Am Chem Soc 1986; 108:5771-5779.

Doi RH, Kosugi A: Cellulosomes: plant-cell-walldegrading enzyme complexes. Nat Rev Microbiol 2004;4:541-551.

Duff M, Borreguero JM, Cuneo M, Ramanathan A, He J, Kamath G, Chennubhotla C, Meilleur F, Howell EE, Herwig KW, Myles DAA, Agarwal PK: Manipulating enzyme efficiency through controlling protein dynamics. Submitted 2017.

Fraser JS, Clarkson MW, Degnan SC, Erion R, Kern D, Alber T: Hidden alternative structures of proline isomerase essential for catalysis. Nature 2009;462:669-673.

Gaffarogullari EC, Greulich P, Kobitski AY, Nierth A, Nienhaus GU, Jaschke A: Unravelling RNA-substrate interactions in a ribozyme-catalysed reaction using fluorescent turn-on probes. Chemistry 2015;21:58645871.

Gibson C, Matthews I, Samuel A: Microwave enhanced diffusion in polymeric materials. J Microwave Power Electromagnetic Energy 1988; 23:17-28.

Hammes-Schiffer S, Benkovic SJ: Relating protein motion to catalysis. Annu Rev Biochem 2006; 75:519-541.

Hammes GG, Benkovic SJ, Hammes-Schiffer S: Flexibility, diversity, and cooperativity: pillars of enzyme catalysis. Biochemistry 2011;50: 10422-10430.
He M, Taussig MJ: Ribosome display: cell-free protein display technology. Brief Funct Genomic Proteomic 2002;1:204-212.

Henzler-Wildman K, Kern D: Dynamic personalities of proteins. Nature 2007;450:964-972.

Hilvert D: Critical analysis of antibody catalysis. Annu Rev Biochem 2000;69:751-793.

Hilvert D, Hill KW, Nared KD, Auditor MTM: Antibody catalysis of a Diels-Alder reaction. J Am Chem Soc 1989;111:9261-9262.

Hu Y, Houk KN, Kikuchi K, Hotta K, Hilvert D: Nonspecific medium effects versus specific group positioning in the antibody and albumin catalysis of the base-promoted ringopening reactions of benzisoxazoles. J Am Chem Soc 2004; 126:8197-8205.

Ingerson-Mahar M, Briegel A, Werner JN, Jensen GJ, Gitai Z: The metabolic enzyme CTP synthase forms cytoskeletal filaments. Nat Cell Biol 2010;12:739-746.

Inoue T, Joyce GF, Grzeskowiak K, Orgel LE, Brown JM, Reese CB: Template-directed synthesis on the pentanucleotide CpCpGpCpC. J Mol Biol 1984;178:669-676.

Kanan MW, Rozenman MM, Sakurai K, Snyder TM, Liu DR: Reaction discovery enabled by DNA-templated synthesis and in vitro selection. Nature 2004;431:545-549.

Kanoatov M, Javaherian S, Krylov SN: Selection of aptamers for a non-DNA binding protein in the context of cell lysate. Anal Chim Acta 2010;681:92-97.

Kelly TR, Bridger GJ, Zhao C: Bisubstrate reaction templates. Examination of the consequences of identical versus different binding sites. J Am Chem Soc 1990;112:8024-8034.

Krylov SN: Kinetic CE: foundation for homogeneous kinetic affinity methods. Electrophoresis 2007;28:69-88.

Lange OF, Lakomek NA, Fares C, Schroder GF, Walter KF, Becker S, Meiler J, Grubmuller H, Griesinger C, de Groot BL: Recognition dynamics up to microseconds revealed from an RDC-derived ubiquitin ensemble in solution. Science 2008;320:1471-1475.

Lechene CP, Luyten Y, McMahon G, Distel DL: Quantitative imaging of nitrogen fixation by individual bacteria within animal cells. Science 2007;317:1563-1566.

Legent G, Delaune A, Norris V, Delcorte A, Gibouin D, Lefebvre F, Misevic G, Thellier M, Ripoll C: Method for macromolecular colocalization using atomic recombination in dynamics SIMS. J Phys Chem B 2008;112:5534-5546.

Manning GS: Counterion condensation on charged spheres, cylinders, and planes. J Phys Chem B 2007;111:8554-8559.

Marti S, Andres J, Moliner V, Silla E, Tunon I, Bertran J: Theoretical study of catalytic efficiency of a Diels-Alderase catalytic antibody: an indirect effect produced during the maturation process. Chemistry 2008;14:596-602.
McHaourab HS, Oh KJ, Fang CJ, Hubbell WL: Conformation of T4 lysozyme in solution. Hinge-bending motion and the substrate-induced conformational transition studied by site-directed spin labeling. Biochemistry 1997;36:307-316.

Musheev MU, Kanoatov M, Krylov SN: Non-uniform velocity of homogeneous DNA in a uniform electric field: consequence of electricfield-induced slow dissociation of highly stable DNA-counterion complexes. J Am Chem Soc 2013;135:8041-8046.

Narayanaswamy R, Levy M, Tsechansky M, Stovall GM, O'Connell JD, Mirrielees J, Ellington $\mathrm{AD}$, Marcotte EM: Widespread reorganization of metabolic enzymes into reversible assemblies upon nutrient starvation. Proc Natl Acad Sci USA 2009;106:10147-10152.

Naylor R, Gilham PT: Studies on some interactions and reactions of oligonucleotides in aqueous solution. Biochemistry 1966;5:27222728.

Norris V, Amar P, Legent G, Ripoll C, Thellier M, Ovadi J: Sensor potency of the moonlighting enzyme-decorated cytoskeleton: the cytoskeleton as a metabolic sensor. BMC Biochem 2013;14:3.

Norris V, Engel M, Demarty M: Modelling biological systems with competitive coherence. Adv Artif Neural Syst 2012;2012:1-20.

Norris V, Turnock G, Sigee D: The Escherichia coli enzoskeleton. Mol Microbiol 1996;19: 197-204.

Norris V, Zemirline A, Amar P, Audinot JN, Ballet P, Ben-Jacob E, Bernot G, Beslon G, Cabin A, Fanchon E, Giavitto JL, Glade N, Greussay P, Grondin Y, Foster JA, Hutzler G, Jost J, Kepes F, Michel O, Molina F, Signorini J, Stano P, Thierry AR: Computing with bacterial constituents, cells and populations: from bioputing to bactoputing. Theory Biosci 2011; 130:211-228.

Oosawa F: Polyelectrolytes. New York, Dekker, 1971.

Paige JS, Nguyen-Duc T, Song W, Jaffrey SR: Fluorescence imaging of cellular metabolites with RNA. Science 2012;335:1194.

Peters KS, Snyder GJ: Time-resolved photoacoustic calorimetry: probing the energetics and dynamics of fast chemical and biochemical reactions. Science 1988;241:1053-1057.

Pollack GH: The Fourth Phase of Water: Beyond Solid, Liquid, and Vapor. Seattle, Ebner and Sons, 2013.

Preiswerk N, Beck T, Schulz JD, Milovnik P, Mayer C, Siegel JB, Baker D, Hilvert D: Impact of scaffold rigidity on the design and evolution of an artificial Diels-Alderase. Proc Natl Acad Sci USA 2014;111:8013-8018.

Ramanathan A, Agarwal PK: Computational identification of slow conformational fluctuations in proteins. J Phys Chem B 2009;113: 16669-16680.

Ramanathan A, Savol A, Burger V, Chennubhotla CS, Agarwal PK: Protein conformational populations and functionally relevant substates. Acc Chem Res 2014;47:149-156. 
Riedel C, Gabizon R, Wilson CA, Hamadani K, Tsekouras K, Marqusee S, Presse S, Bustamante C: The heat released during catalytic turnover enhances the diffusion of an enzyme. Nature 2015;517:227-230.

Ripoll C, Norris V, Thellier M: Ion condensation and signal transduction. Bioessays 2004;26: 549-557.

Romesberg FE, Spiller B, Schultz PG, Stevens RC: Immunological origins of binding and catalysis in a Diels-Alderase antibody. Science 1998; 279:1929-1933.

Rothlisberger D, Khersonsky O, Wollacott AM, Jiang L, DeChancie J, Betker J, Gallaher JL, Althoff EA, Zanghellini A, Dym O, Albeck S, Houk KN, Tawfik DS, Baker D: Kemp elimination catalysts by computational enzyme design. Nature 2008;453:190-195.

Shah S, Gupta MN: The effect of ultrasonic pretreatment on the catalytic activity of lipases in aqueous and non-aqueous media. Chem Cent J 2008;2:1.

Sharma S, Kumar SK, Buldyrev SV, Debenedetti PG, Rossky PJ, Stanley HE: A coarse-grained protein model in a water-like solvent. Sci Rep 2013;3:1841.

Siegel JB, Zanghellini A, Lovick HM, Kiss G, Lambert AR, St Clair JL, Gallaher JL, Hilvert D, Gelb MH, Stoddard BL, Houk KN, Michael FE, Baker D: Computational design of an enzyme catalyst for a stereoselective bimolecular Diels-Alder reaction. Science 2010;329:309313.
Smith GP: Filamentous fusion phage: novel expression vectors that display cloned antigens on the virion surface. Science 1985;228:13151317.

Srere P: Complexities of metabolic regulation. Trends Biochem Sci 1994;19:519-520.

Stanic-Vucinic D, Stojadinovic M, AtanaskovicMarkovic M, Ognjenovic J, Gronlund H, van Hage M, Lantto R, Sancho AI, Velickovic TC: Structural changes and allergenic properties of beta-lactoglobulin upon exposure to highintensity ultrasound. Mol Nutr Food Res 2012;56:1894-1905.

Stewart JD, Krebs JF, Siuzdak G, Berdis AJ, Smithrud DB, Benkovic SJ: Dissection of an antibody-catalyzed reaction. Proc Natl Acad Sci USA 1994;91:7404-7409.

Strogatz SH, Stewart I: Coupled oscillators and biological synchronization. Sci Am 1993;269: 102-109.

Sytina OA, Heyes DJ, Hunter CN, Alexandre MT, van Stokkum IH, van Grondelle R, Groot ML: Conformational changes in an ultrafast lightdriven enzyme determine catalytic activity. Nature 2008;456:1001-1004.

Takahashi TT, Austin RJ, Roberts RW: mRNA display: ligand discovery, interaction analysis and beyond. Trends Biochem Sci 2003;28: 159-165.

Tonelli RR, Colli W, Alves MJ: Selection of binding targets in parasites using phage-display and aptamer libraries in vivo and in vitro. Front Immunol 2012;3:419.

Volker J, Breslauer KJ: Communication between noncontacting macromolecules. Annu Rev Biophys Biomol Struct 2005;34:21-42.
Volkov SN, Kosevich AM: Theory of low-frequency vibrations in DNA macromolecules. J Biomol Struct Dyn 1991;8:1069-1083.

von Kiedrowski G: A self-replicating hexadeoxynucleotide. Angew Chem Int Ed 1986;25: 932-935.

Wang SC, Lee CT Jr: Enhanced enzymatic activity through photoreversible conformational changes. Biochemistry 2007;46:14557-14566.

Weart RB, Lee AH, Chien AC, Haeusser DP, Hill NS, Levin PA: A metabolic sensor governing cell size in bacteria. Cell 2007;130:335-347.

Wegman DW, Cherney LT, Yousef GM, Krylov SN: Universal drag tag for direct quantitative analysis of multiple microRNAs. Anal Chem 2013;85:6518-6523.

Yonezawa M, Doi N, Higashinakagawa T, Yanagawa $\mathrm{H}$ : DNA display of biologically active proteins for in vitro protein selection. J Biochem 2004;135:285-288.

Young DD, Nichols J, Kelly RM, Deiters A: Microwave activation of enzymatic catalysis. J Am Chem Soc 2008;130:10048-10049.

Yufa R, Krylova SM, Bruce C, Bagg EA, Schofield CJ, Krylov SN: Emulsion PCR significantly improves nonequilibrium capillary electrophoresis of equilibrium mixtures-based aptamer selection: allowing for efficient and rapid selection of aptamer to unmodified ABH2 protein. Anal Chem 2015;87:14111419.

Zheng F, Zhan CG: Structure-and-mechanismbased design and discovery of therapeutics for cocaine overdose and addiction. Org Biomol Chem 2008;6:836-843. 